ican Indians, as well as those of Mexico and Peru. Sometimes a board was bound against the forehead of an infant and kept there during early growth, resulting in a hideous flatness of the front of the cranium (Flathead Indians). Other tribes, such as the Vancouvers, Incas and Aztecs, applied pads and tight bandages to the head in such ways as to render the skull conical, enormously elongated, or deformed in other monstrous ways. Although producing an outward semblance of idiocy, these peculiarities of form did not seem in any way to influence the mentality. "Flattening of the occipital region, so commonly observed in collections of prehistoric skulls, was probably more or less accidental and due to pressure of the infants' skulls against the boards on which they were habitually strapped and carried.

Anesthetics.-Although it cannot be questioned that some of our primitive peoples possessed more or less reliable methods of anesthesia, it is hardly probable that these were very generally known or employed. For instance, the Zunis and some other tribes used for the purpose a substance obtained from the jimson weed (Datura meteloides), containing stramonium. It was administered in sufficient amount to produce indifference to pain or even complete unconsciousness, and in this condition abscesses were opened, fractures set, dislocations reduced, and other surgical procedures accomplished. In spite of heroic dosage, no serious harm seemed to result. It is quite possible that this and similar methods were also in use among the Aztecs and Incas, who were so closely related in many ways to the Pueblos.

In this connection should not be overlooked the strong hypnotic influence undoubtedly exercised by the medicine-men, with their bizarre make-ups, weird incantations, and fantastic antics, which were well calculated to make a profound impression on their credulous patients.

Metropolitan Building.

Housing Conditions and War Workers.-The necessity for adequate and better housing conditions for men and women workers in the war industries in this country has arisen in an acute form, not only in towns and cities of considerable size where these industries are located, but also in localities where munitions plants, for instance, have been built remote from places of sufficient size to afford any possibility of housing the employees. It has been the experience of England since the beginning of the war that the usual agencies for providing housing for industrial workers have fallen down, in that greater returns on capital have been possible from other sorts of investment. After experimenting with various plans of encouraging the building of houses, including loans of increasing proportions of the capital required, the government has been compelled to assume completely the job of providing houses. At first, cottages or hutments of a more or less temporary nature, community cottages and barracks were constructed, but the experience has culminated in the planning of complete housing communities and the erection of permanent houses of a very good and substantial class, with wide streets, pleasant outlook, good sized gardens, and the conveniences and comforts of modern, healthful ways of living. A writer in the Journal of the American Institute of Architects has described the evolution of the British government housing problem during wartime, and while he feels that in this country the problem is not identical, and that the whole matter should be studied from a different point of view, yet he reluctantly concludes that "if English experience has any value at all for us in this matter, it seems clearly to point in the direction of the nation itself shouldering a burden which has become too heavy to be carried by employers and individual communities alone."

\title{
OBSTRUCTIVE STERILITY IN THE MALE
}

TREATMENT BY DIRECT VASO-EPIDIDYMOSTOMY *

V. D. LESPINASSE, M.D.

Assistant Professor of Genito-Urinary Surgery, Northwestern University School of Medicine

\section{CHICAGO}

Obstructive sterility in the male is easily diagnosed by microscopic examination of the semen. The semen shows a complete absence of spermatozoa. The only treatment in these cases is surgery. We must make an anastomosis around the obstruction to permit the passage of the sperm. The particular operation necessary to be performed depends on the location of the obstruction, thus :

1. If the obstruction is in the epididymis, the operation will be direct vaso-epididymostomy.

- 2. In the scrotal vas, resection of the obstruction and union of the vas, either end to end or lateral.

3 . In the pelvic vas or ejaculatory duct, author's sac operation.

4. In the pelvic vas on one side and epididymis or scrotal vas on the other side, anastomosis of the vas

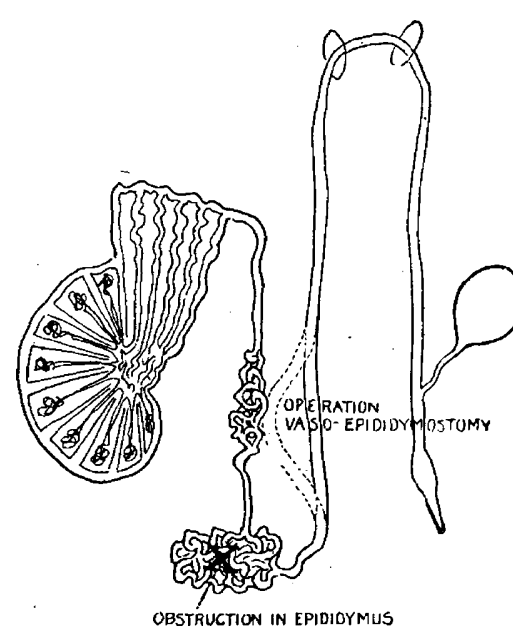

Fig. 1.- Schematic drawing of the sexual tract of the male; $x$ marks an obstruction in the tail of the epididymis, and the opera tion necessary for relief, direct vaso-epi line.

\section{tral end of the}

opened vas and noting its appearance or nonappearance in the urethra.

When the obstruction is found in the globus minor of the epididymis, the operation necessary to relieve this obstruction is an anastomosis between the epididymis tubule (Fig. 1) above the point of obstruction and the vas deferens below any obstruction that may be present in it. All the present operations (Martin's operation) designed to join the vas to the epididymis tubule are indirect; that is, after the epididymis capsule has been incised, a small portion of the protruding epididymis tubule is cut off, then the vas is split longitudinally, and the open incision in the vas is sewed to the epididymis capsule over the raw surface of the epididymis; hence there is a considerable distance that must be bridged by epithelium between the functional end of the epididymis tube and the vas. Unfortunately, this bridge often fails to develop, and consequently the operation is a failure.

* Awarded silver medal, Scientific Exhibit, Sixty-Eighth Annua Session of the American Medical Association, New York, June, 1917. * From the Laboratory of Experimental Surgery, Northwestern University Medical School. 
The two chief causes of failure are (1) the overgrowth of connective tissue, choking the epididymis tubule, and (2) the organizing of the blood clot that forms on the cut surface of the epididymis, whereby the epididymis tubule is closed. To circumvent these difficulties I devised an operation for direct anastomosis between the epididymis tubule and the lumen of the vas. I have called this operation direct vaso-
In Figure 4 we see the needle drawn through, and the outpouring of the epididymal secretion. This secretion is aspirated into a small syringe, and then placed on a microscopic slide and examined for spermatozoa. The ends of this crown or epididymal stitch are now passed through the longitudinal incision into the vas, down the lumen of the vas, and out through the wall of the vas. When both ends of the crown

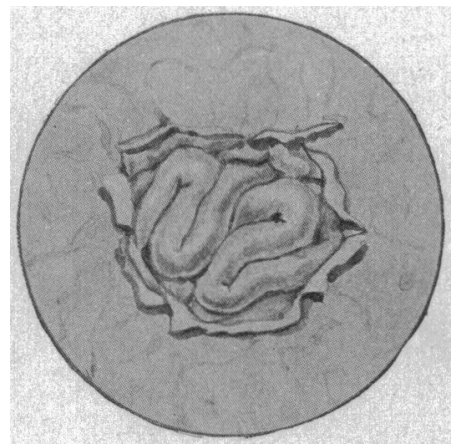

Fig. 2.-Capsule of the epididymis cut through and reflected, exposing the epididymis tubule characteris. the epididymis tubule

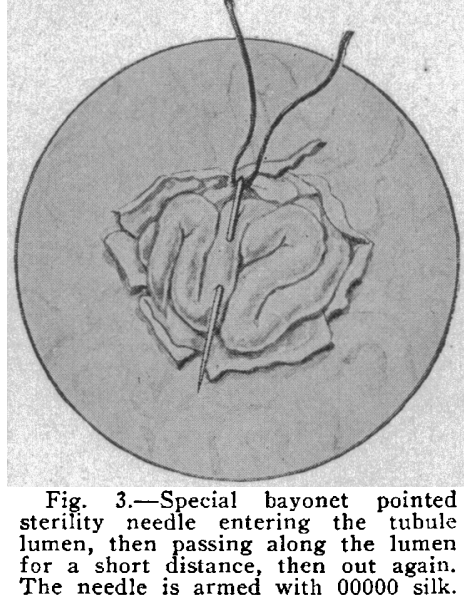

epididymostomy. As it brings the epithelium of the vas into direct union with the epithelial lining of the epididymis tubule, the danger of hemorrhage is absolutely eliminated, and the danger of choking by connective tissue is practically eliminated.

TECHNIC OF DIRECT VASO-EPIDIDYMOSTOMY

An incision is made into the scrotum down to and into the tunica vaginalis testis. The epididymis is exposed, and the point of obstruction is noted. The lumen of the vas is now opened by a short longitudinal incision, and a colored fluid is injected into the central end of the vas. If the fluid appears in the urethra, we know positively that the vas is open from the point of incision to the urethra. Next, the epididymis capsule over the epididymis compartment immediately above the obstruction is incised carefully down to the tubules, great care being taken (1) that all the different layers of the capsule are removed from the epididymis tubule, and (2) that the epididymis tubule itself is not nicked or cut into at any point. The epididymis tubule will protrude through the opening thus made in the epididymis capsule (Fig. 2). A tubule is now selected whose direction is the same as the long axis of the body of the epididymis.

A suture of 00000 silk mounted on a No. 19 bayonet pointed needle is now passed through the wall of the epididymis tubule (Fig. 3 ), then down the lumen of the tubule and out again through the wall of the epididymis tubule, from 2 to $4 \mathrm{~mm}$. from its point of entrance (Figs. 3, 4 and 5). suture have been inserted into their appropriate ends of the vas incision, the appearance is as shown in Figure 6.

Sutures of extraordinarily fine catgut, made especially for this purpose by Mr. Hollister, are now passed, one on each side of the longitudinal incision in the vas. These sutures (Fig. $7 C$ and $D$ ) includes both the wall and the epithelial lining of the vas in

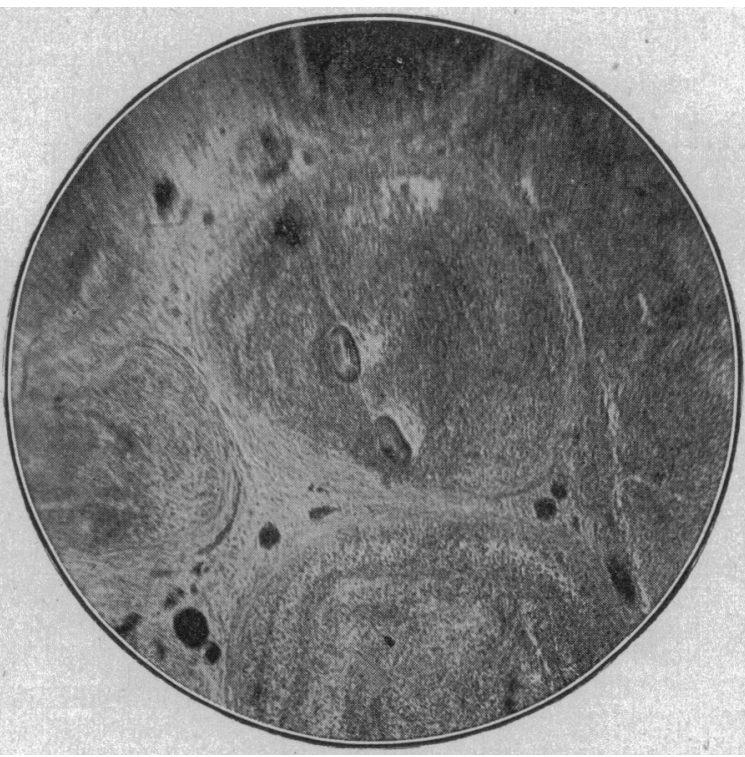

Fig. 5.-Low power photomicrograph of the epididymis tubule into whose lumen has been passed a suture of human hair. The two oval bodies are the cross-section of the hair. its grasp. These sutures are designed to hold the incision in the vas open. Sutures (Fig. $7 A$ and $B$ ) are now passed into the wall of the vas a short distance from each end of the incision. These sutures include only the wall of the vas, and do not enter the lumen of the vas at all. They are designed to fix the vas to the epididymis and carry the weight of the testicle, and are so spaced on the length of the vas that when they are tied down they leave the intervening vas slack, so as to remove all tension from the union between the epididymis tubule and the vas.

When the four sustaining sutures are passed and the vas is turned over, the appearance is as shown in Figure 7 . The central or upper end of the crown stitch is now threaded on a long Hagedorn needle and after the testicle has been replaced into the scrotum, this needle transfixes the scrotum from inside out, bringing the crown thread with it. 'In from one to two weeks, when a necrosis of the wall of the epididymis tubule which has been in the grasp of the suture has taken place, this suture can be removed by slow gradual traction. 
In order to visualize the amount of material that passes through one of these anastomoses, the spermatozoa were recovered from the entire amount of semen ejaculated. This has been done from different individuals and from the same individual at different times. The amount varies with the vigor and passion of the intercourse. A large lumen is not necessary. The size of the lumen obtained by this operation is consider this material diagnostic of a vas that has been obstructed at least ten years.

After this technic was fully developed, I performed direct vaso-epididymostomy on one side only of eleven dogs. These animals were killed two months after the operation. The vas of the side not operated on was stripped, and spermatozoa were demonstrated in each instance. The vas coming from the epididymis

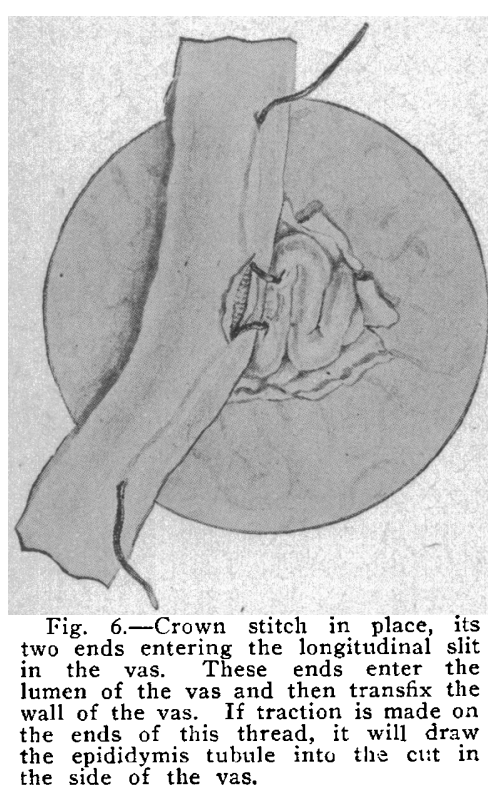

shown in Figure 8, a specimen on which the operation of direct vaso-epididymostomy had been performed two months before. The vas has been torn from the epididymis, and in the center of the vas can be seen the little opening that connected the vas lumen with the epididymis tubule.

The anastomosis made by the operation is illustrated in Figures 9, 10 and 11.

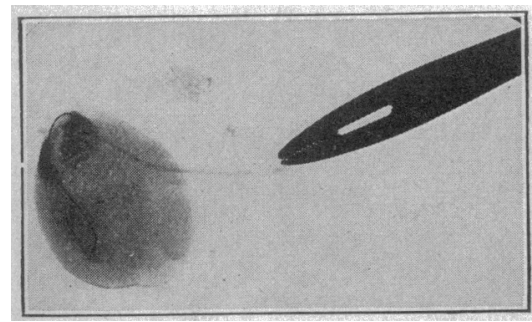

Fig. 9.- Injected vas and injection in first compartment of epididymis tubule, demonstrating that the anastomosis is patent. The crown stitch in this instance was of silver wire.

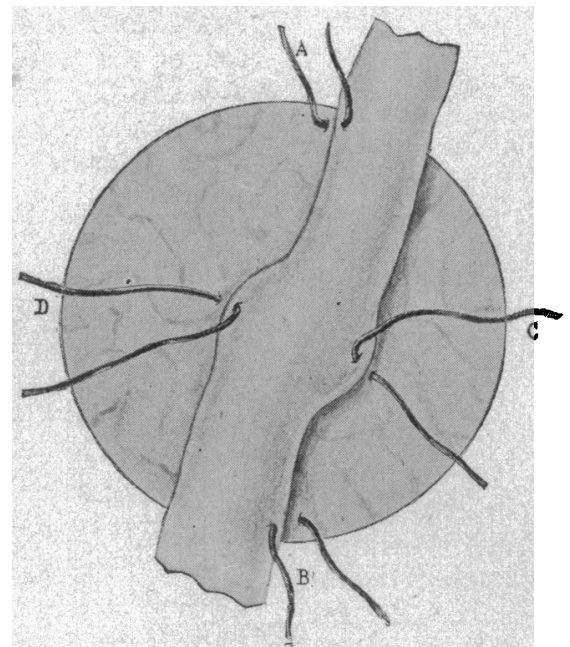

Fig. 7,-Two stitches, $A$ and $B$, which fix the vas to the capsule of the epididymis and carry the weight of the testicle. They are so placed that the vas between them is hold open the longitudinal slit in the vas.

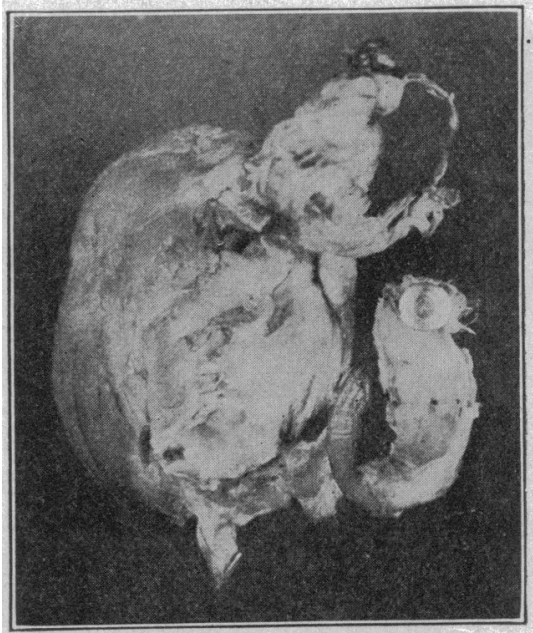

Fig. 8.-Vas on which the operation of direct vaso.epididymostomy had been performed two months previously, torn from the epididymis and viewed from the epididymal epididymis and viewed from the epididymal in the center of the picture.
Usually when we incise the vas we find that the lumen is empty. Occasionally we find that it is filled. with a thick creamy material which, on examination, is found to be composed practically entirely of spermatozoa. In three instances in which the vas had been occluded for many years, the vas was filled with this creamy material; on examination it was found to contain no spermatozoa whatsoever, but simply round amorphous material which had the microscopic appearance of fat globules. They do not take the fat stain, however, and their exact chemical composition is not certain. In all of the cases in which this material has been found, the vas was obstructed in the pelvis. I operated on was similarly stripped, and spermatozoa were demonstrated in ten of the eleven operations. The patency of the anastomosis is shown by the epididymograms (Figs. 9, 10 and 11).

\section{REPORT OF CASE}

A patient with bilateral epididymitis showed slight thickenings in the globus minor of both epididymides, and absence of spermatozoa from the semen. The left testicle was explored under local anes-

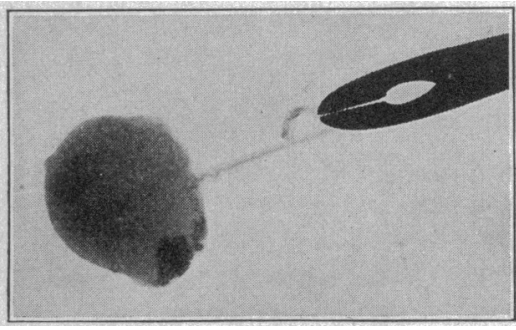

Fig. 11.-Exact location and size of the connecting lumen between the vas and the epididymis tubule. The crown stitch is of silk, and consequently it does not show. 